\title{
Condiciones hidrológicas y su relación con la pobreza en Colombia: un análisis desde el ámbito departamental ${ }^{*}$
}

\section{Relationship between Hydrological \\ Conditions and Poverty}

in Colombia: A regional Analysis

Rubén Darío Díaz Mateus** Edisson Stiven Castro Escobar****

Recibido: 1 de Junio de 2018

Revisado: 27 de Agosto de 2018

Aprobado: 15 de Septiembre de 2018

\section{Resumen}

En la presente investigación se analiza la relación entre las condiciones hidrológicas y la pobreza de Colombia a nivel departamental, mediante un conjunto de variables explicativas y datos a nivel territorial con los que se estimó un modelo de regresión a

* Artículo de investigación producto del proyecto denominado Hidrología y pobreza un análisis departamental. Cómo citar este artículo: Mateus, R. D., y Castro, E. E. (2019). Condiciones hidrológicas y su relación con la pobreza en Colombia: un análisis desde el ámbito departamental. Revista CIFE: Lecturas de Economía Social, 20(32), 51-77. doi: https://doi.org/10.15332/22484914.4856

** Economista. Magíster en Desarrollo Sostenible y Medio Ambiente de la Universidad de Manizales. Docente- investigador de la Universidad de La Salle. Correo electrónico: rudiaz@unisalle.edu.co

*** Economista. Magíster en Economía de la Universidad de Manizales. Correo electrónico: ecastro@umanizales.edu.co 
dos niveles de corte transversal, incluyendo como variable respuesta el indicador de Incidencia de Pobreza Monetaria y como variables explicativas indicadores socioeconómicos e hidrológicos de los departamentos. Las relaciones entre las condiciones hidrológicas y la pobreza a nivel departamental permiten evidenciar una asociación positiva entre el Índice de Riesgo de la Calidad de Agua (IRCA) y la pobreza monetaria, esto indica que los departamentos con mejor calidad de agua tienen un nivel menor de pobreza que los departamentos con un IRCA alto. Los resultados manifiestan la necesidad de integrar acciones que consideren las condiciones de oferta y demanda hídrica en los territorios como política social.

Palabras clave: agua, pobreza, calidad de agua.

Clasificación JEL: Q25, I3

\section{Abstract}

The relationship between hydrological conditions and Colombia's poverty were analyzed through a statistical approach with territorial data and a cross-sectional regression model, which considerate the correlation indicator between Monetary Poverty and hydrological indicators of the regions. This model shows a positive association between the Water Quality Risk Index (IRCA) and the monetary poverty, which indicates that the departments with high water quality have a lower poverty level. The results indicate the need to integrate the social policies with the conditions of water offer and demand in the territories.

Keywords: Water, poverty, water quality.

Classification JEL: Q25, I3 


\section{Introducción}

Las condiciones de desarrollo económico en Colombia han estado al margen de consideraciones estructurales como la capacidad de carga de los ecosistemas, en esa vía la frontera de posibilidades de producción ha sido condicionada por la dotación de factores básicos como el capital y el trabajo, sin considerar las limitaciones en el stock de la oferta ambiental, esto se evidencia en la relación negativa que persiste entre la degradación de los recursos naturales y el crecimiento económico. Así mismo, el deterioro ambiental ha sido un determinante del aumento de desigualdades sociales y la marginalización de regiones sometidas directamente a los daños colaterales en la afectación ambiental, tales como el cambio climático, la contaminación hídrica, la alteración de los usos del suelo, la presión atmosférica y la pérdida de biodiversidad. En este contexto, la investigación pretende interpretar la relación entre las condiciones hidrológicas como la cantidad y calidad del agua como determinante de pobreza en el ámbito territorial, para determinar la vulnerabilidad de la población en Colombia a partir de indicadores biofísicos e información socioeconómica.

Tradicionalmente la pobreza ha sido estudiada desde la perspectiva de los ingresos monetarios sin interpretar otras realidades territoriales. La propuesta multidimensional ha sentado nuevas bases como un hecho que se determina además por las capacidades sociales, las libertades individuales y la provisión de servicios básicos. De todos modos, estas nuevas interpretaciones siguen siendo muy limitadas respecto al entorno socioambiental y funcional que tiene los ecosistemas como fuentes de bienes y servicios, materia energética, control biológico y sobre todo como soporte a la vida, por ejemplo, en aspectos como las condiciones de calidad y cantidad de agua, la temperatura, la cubierta boscosa y la superficie de suelo con cobertura vegetal, entre otras. Por ello, en la perspectiva de desarrollo del país y en el marco de los objetivos de desarrollo sostenible, la reducción de la pobreza considera necesario que su erradicación se garantice mediante el aumento del acceso a servicios básicos. En los Objetivos de Desarrollo del Milenio (ODM) en Colombia había una meta de aumentar la cobertura de agua potable y los esquemas de saneamiento básico, y aunque hubo resultados favorables, se estima que aún hay un porcentaje importante del territorio nacional con problemas básicos de infraestructura para la provisión de esos servicios (para 2015 la cobertura de agua potable era de $73.8 \%$ y de saneamiento básico del $67.9 \%$ ) (Carrasco, 2016).

Según Feres y Mancero (2001), citados en Abadí y Vitale (2015), los estudios económicos de pobreza hacen énfasis en palabras como necesidad, estándar de vida e insuficiencia de recursos, pero en las opciones aceptadas de medición solo están la satisfacción de necesidades, el consumo de bienes y el ingreso disponible, es decir, apunta a una cuantificación del bienestar de los individuos. Por tanto, la desigualdad y el crecimiento 
económico se convierten en un factor relacionado con la pobreza, pero esta a su vez con las privaciones sociales en materia de servicios básicos ambientales.

En América Latina cerca de 160 millones de personas (28 \% de su población) siguen viviendo en la pobreza, donde uno de los factores más influyentes de esta condición tiene que ver con la falta de acceso a servicios básicos, y aunque sin duda se han mejorado los niveles de abastecimiento de agua potable y saneamiento, aún se presentan graves deficiencias en la calidad del servicio que afecta en diferente medida las áreas rurales y a la población más pobre (Jouravlev, 2011, citado en Unesco, 2015, p. 10). Según el DANE (2016), la pobreza monetaria en Colombia para el año 2015 fue del $27.8 \%$ con una proporción de pobreza extrema de $7.9 \%$. Igualmente, la pobreza multidimensional a nivel nacional representó el $21.9 \%$, y en términos de desigualdad el índice de Gini registró 0,522 en el 2015.

Este panorama es más grave cuando se realiza el análisis por departamentos. En general se evidencia mayor pobreza monetaria para el 2015 en Chocó con 62.8 \%, La Guajira con $53.3 \%$ y Cauca con $51.6 \%$; al tiempo que la mayor pobreza extrema se presentó en estos mismos departamentos (Chocó con $37.1 \%$, La Guajira con $24.3 \%$ y Cauca con $24.0 \%$ ) (DANE, 2016a). Ante el panorama anterior y asumiendo que la pobreza en Colombia por regiones está determinada por las dificultades en términos físicos de las áreas hidrológicas, se pretende en la investigación reconocer cuáles son las relaciones entre la cantidad y calidad del agua y la pobreza en el ámbito departamental en el periodo 2016. Se parte del argumento que un estado crítico de los ecosistemas y las microcuencas hidrológicas se constituye un determinante clave de pobreza en Colombia.

\section{Análisis de la pobreza desde una aproximación multidimensional}

El concepto de pobreza postulado por el Banco Mundial (1990, pp. 26-27) se entiende como "la incapacidad que la gente tiene de obtener un nivel mínimo de vida. Esta definición es incompleta ya que implica elementos más profundos" (Ortiz y Ríos, 2013, p. 190). La clasificación de la pobreza también ha sido establecida como factor de subdesarrollo en una economía (Ray, 1999), donde habría menores y volátiles ingresos y por consiguiente mayor analfabetismo, desnutrición, inestabilidad en la salud, entre otros efectos sociales que inciden en la cotidianidad de los individuos. En ese sentido, Domínguez y Martínez (2006), citados en Ortiz y Ríos (2013, p. 195), relacionan la pobreza con "la incapacidad de las personas de vivir una vida tolerable", donde los pobres son cada vez más vulnerables a restricciones externas. 
Tradicionalmente el concepto de pobreza se ha establecido en función de un parámetro inferior de ingresos al conjunto promedio de las familias, que influye no solo en la privación de condiciones materiales, sino de los niveles de vida, las costumbres y los hábitos de gozo de toda la sociedad (Townsend y Gordon, 1989; Gordon y Townsend, 2000; Gordon y Pantazis, 1997; Veit, 1998, citado en Spicker, Álvarez y Gordon, 2009). En esa perspectiva, la pobreza al estar centrada en una medición unidimensional con la característica de un ingreso mínimo que influye en el bienestar humano, tiende a percibir un límite en los ingresos que a su vez limitarán el acceso a bienes o de activos específicos que conllevan a la privación de capacidades de un individuo, rasgo explicativo en la literatura científica dominante (Rodríguez y Sánchez, 2017, p. 144). De ahí que la pobreza solo hace visible al sujeto por su capacidad adquisitiva de bienes y servicios básicos.

La imagen parcial de la pobreza desde los ingresos no permite incorporar las otras afectaciones de la vida humana desde la cotidianidad de un individuo, por tanto, un individuo puede tener ventajas en salud, pero ser analfabeta y ser excluido en la gestión de conocimiento y comunicación o en la condición contraría donde su conocimiento, no lo hace inmune a una condición epidemiológica de vulnerabilidad. Bajo las consideraciones anteriores, la pobreza ha sido entendida entonces desde dos grandes criterios, uno objetivo y otro subjetivo. El primero se caracteriza por estudiar el contexto de subsistencia y necesidades básicas limitado a la forma de vida del individuo y su hogar, es decir, se es pobre si el ingreso del hogar no cubre los satisfactores básicos mínimos para establecer una condición fisica aceptable; como tal definición incorpora satisfactores básicos, el análisis profundiza en las necesidades vitales donde se incluyen los servicios públicos, salud y educación (Pinzón, 2017, p. 14). De ahí que el estudio de la pobreza se asocia a parámetros monetarios y no monetarios, pero con gran énfasis en los impactos que genera la pérdida de ingresos en el hogar o individuo. El segundo se refiere a aspectos cognitivos sobre la percepción de bienestar.

Según Ravallion, Shaohua y Prem (2009), citado en el Consejo Nacional de Evaluación de la Política de Desarrollo Social (2014), el enfoque unidimensional compara las condiciones de vida, referenciando el indicador de línea de pobreza donde señala que una persona es pobre si vive con 1.25 dólares estadunidenses por día (ajustados por la paridad del poder adquisitivo). Estas son mediciones que no consideran el valor intrínseco del ser humano y las realidades no monetarias en las transacciones, de todos modos, los avances de investigación en la relación con la pobreza y el desarrollo humano han aumentado para incorporar los análisis de variables satisfactorias y las características propias de un individuos como de su entorno natural y soportes de vida (Townsend, 1979; Sen, 2000; Deaton, 2001; Ferrer-i-Carbonell, 2002; Ravallion, 2011, citado en Pinzón, 2017, p. 13). 
Bajo esa consideración se ha previsto como único camino para superar la pobreza los mayores niveles de crecimiento económico desde este enfoque de objetividad. Según Rodrik (2012), citado en García (2016, p. 72) "la globalización puede solucionar estos problemas, dando acceso a los mercados, al capital, a las nuevas tecnologías y mejorando de esta manera los niveles de pobreza", de lo anterior, se sintetiza que a mayores grados de apertura comercial en las economías, se genera una incidencia en los niveles de ingreso y estos vinculan un efecto positivo con los niveles de pobreza, supuesto que deja implícito el crecimiento económico como garantía de "eliminar la persistencia de la pobreza relativa, como parece ocurrir con la pobreza absoluta cuando se cae en "trampas de pobreza"”' (Sachs, 2005; Ros, 2004; Griffin, 1989, citado en Macías, 2014, p. 118).

Así mismo, la visión tradicional condiciona el análisis a una dicotomía de ser pobre o no según el desempeño de indicadores como: el crecimiento económico, mercado laboral, rol de instituciones, cambio demográfico, niveles educativos, ingreso no laboral entre otros, además existen una escala diferenciadora en el contexto geográfico y regional, es decir, la pobreza rural es más elevada, intensa y permanente frente a la pobreza urbana, por tanto, esta heterogeneidad también incide en los aspectos de cambio de la pobreza (Sánchez, 2015). Esta influencia no solo se da desde el enfoque unidimensional, sino que incide en el enfoque multidimensional del bienestar al señalar aspectos como las condiciones de trabajo, el tipo de inserción laboral y niveles de dependencia económica (Alkire y Foster, 2009). No obstante, otra lectura del crecimiento económico y pobreza, la presenta Ravallion (2001), citado en Sánchez (2015, p. 364), al suponer que "un alto aumento de los ingresos medios puede ser contrarrestado por aumentos moderados de la desigualdad en el propósito de reducir la pobreza".

Una posición que ha buscado superar esa visión parcializada de la pobreza ha sido el enfoque multidimensional ${ }^{1}$, que permite un análisis diferenciador de la pobreza desde una perspectiva territorial. El enfoque multidimensional considera una situación de pobreza del individuo que se expone a una o más carencias sociales, adicionalmente los ingresos no soportan una adquisición material satisfactoria frente a las necesidades básicas (Consejo Nacional de Evaluación de la Política de Desarrollo Social, 2014, p. 19). En ese sentido, la perspectiva de la pobreza se ha entendido por aspectos cognitivos sobre la percepción de bienestar, desarrollo de capacidades y libertades. Esta consideración es presentada por Sen (2000) al describir el ser humano con capacidades intrínsecas que hacen posible cambiar su percepción de ser y estar en un territorio como agente de construcción social y ecológica de bienestar. De ahí que el estudio de la pobreza se asocie

1 Entre los enfoques multidimensionales para medir la pobreza se encuentran "el método de las capacidades (Sen, 1993, 1999), las metodologías que combinan el ingreso con las necesidades básicas insatisfechas (NBI) (Beccaria y Minujín, 1985; Feres y Mancero, 2000), el método de medición integrado de la pobreza (MMIP) (Boltvinik, 1992), el enfoque de derechos (Donald y Mottershaw, 2009), el método de privación relativa (Townsend, 1979; Gordon, 2010), la perspectiva de igualdad de oportunidades (Roemer, 1998), los métodos orientados por axiomas (Alkire y Foster, 2007, 2011), entre otros" (Consejo Nacional de Evaluación de la Política de Desarrollo Social, 2014). 
a necesidades básicas y privaciones relativas con parámetros no monetarios para el reconocimiento de la problemática social (Sen, 1980, 1985a, 1985b, 1987, 1992, 1993, citado en Consejo Nacional de Evaluación de la Política de Desarrollo Social, 2014, p. 7). En consecuencia, este enfoque incorpora el objetivo de superar el utilitarismo previsto de la pobreza desde los ingresos para complementar la medición con indicadores desagregados por la condición territorial (González, 2011).

El factor multidimensional como objeto de estudio centra a la pobreza desde la privación social, término trabajado por el Programa de Naciones Unidas para el Desarrollo - PNUD (Giménez y Valente, 2016), el cual se construye conceptualmente y es institucionalizado en la publicación del informe sobre desarrollo humano de 1997, para hacer referencia a una pobreza humana en la perspectiva del desarrollo humano. La pobreza humana está constituida por progresos y privaciones bajo condiciones de desigualdad entre regiones, países, ricos, pobres, mujeres y hombres, de este modo, las privaciones de capacidades hacen referencia a: "años de vida, salud, vivienda, conocimientos, participación, seguridad personal y medio ambiente. Cuando se combinan estos diferentes tipos de privación limitan gravemente las opciones humanas" (Programa de Naciones Unidas para el Desarrollo - PNUD, 1997, p. 32). Posteriormente, el Programa de las Naciones Unidas para el Desarrollo - PNUD, en el Informe sobre Desarrollo Humano de 2010 (PNUD, 2010), sustituye la medición de pobreza del índice de pobreza humana (IPH) por el índice de pobreza multidimensional (IPM), incorporando dimensiones relativas de educación, salud y calidad de vida (Alkire y Santos, 2010, citados en Consejo Nacional de Evaluación de la Política de Desarrollo Social, 2014, p. 12).

En esta concepción una de las privaciones básicas es por el medio ambiente y el fundamento del bienestar, de manera que "el continuo deterioro ambiental es una fuente de empobrecimiento permanente" (Programa de Naciones Unidas para el Desarrollo, PNUD, 1997, p. 37). El desarrollo conceptual en esta vía ciertamente concuerda con lo desarrollado en el informe de la Comisión Sarkozy, integrado por Stiglit, Sen y Fitoussi (2010), donde promulgan el estudio de una economía desde lo multidimensional para involucrar las transformaciones del presente y futuro. Este planteamiento sugiere que la pobreza se estructura a partir de:

El estándar de vida material (ingreso, consumo y riqueza), la salud, la educación, las actividades personales incluido el trabajo, la participación política, las conexiones y las relaciones sociales, el medio ambiente (las condiciones presentes y futuras), la seguridad frente a fenómenos económicos y crisis naturales. (González, 2011, p. 90)

La dependencia del ser humano de los recursos naturales crea un escenario de vulnerabilidad para los individuos pobres, máxime cuando "500 millones de pobres de países en desarrollo viven en regiones ecológicamente frágiles” (Programa de Naciones Unidas para el Desarrollo, PNUD, 1997, p. 32). El resultado de esta vulnerabilidad es el efecto que puede generar los niveles de contaminación en la salud y por doble vía en otra 
privación básica generadora de pobreza, por lo cual "los pobres son más propensos a obtener una distribución desigual de recursos escasos, en este caso, el agua, aumentando así su vulnerabilidad" (Guevara y Lara, 2015, p. 466). El discurso de desarrollo humano permite entonces entender y ejercer una regulación de la pobreza por medio de un fomento en las capacidades individuales o colectivas de la población pobre, como de las oportunidades que ofrece la realidad socioeconómica y ambiental en la que esta vive (Offe 1990; Cortés y Marshall 1993; Fleury 1997; Soldano y Andrenacci 2006; Falappa y Andrenacci 2009; Danani y Hintze 2011, citado en Campana, 2014, p. 83). En otras palabras, las necesidades de los individuos dependen de las posibilidades de cambios en sus entornos sociales, económicos, políticos y ambientales, con lo cual la posibilidad de acceso a servicios públicos, no es exclusivamente necesario, sino que depende también de la calidad y disponibilidad de servicios eco-sistémicos como soporte de vida que adquiere en la naturaleza humana, en ese sentido por ejemplo, "la relación agua-pobreza es de mayor importancia tanto desde el punto de vista de salud pública como el de equidad social" (Guevara y Lara, 2015, p. 464).

Para Rivera (2012), gran parte de los pobres enfrentan riesgos e incertidumbre por las características de las actividades económicas, debilidades físicas y características del entorno o territorio, más aún, en esta última, si están ubicados en entornos rurales expuestos a variabilidad del clima, cuya incidencia significativa impacta en la frágil condición de salud propensa a enfermedades en población vulnerable como los niños y adultos mayores. De esta manera, la preferencia de bienes primarios que un individuo incorpora en su racionalidad para satisfacer su estilo de vida, está en la cantidad de bienes primarios que incluye la dotación primaria de recursos naturales como soporte de vida de todas las especies en la Biosfera (Pérez, 2011, p. 309). La relación de pobreza y agua no se remite solo a la condición de limitar el acceso a un recurso vital, constituyendo un factor de libertad y desarrollo del individuo, sino a entender que "la privación de este recurso puede limitar que una persona desarrolle plenamente todas sus capacidades, lo cual lo hará más propenso a caer o permanecer en la pobreza" (Guevara y Lara, 2015, p. 466).

\section{Diseño metodológico}

La metodología del estudio corresponde al tipo de investigación descriptivo. Para la validación empírica, se trabajaron indicadores de pobreza como: la incidencia de la pobreza monetaria, incidencia de la pobreza monetaria extrema, coeficiente Gini, promedio del ingreso per cápita de la unidad de gasto de la población, línea de pobreza extrema, brecha de la pobreza monetaria y línea de pobreza; dicha información está representada por las Cifras Departamentales de Pobreza Monetaria y Desigualdad del Departamento Nacional de Estadística (DANE), basada en la Gran Encuesta Integrada 
de Hogares (2008-2016) de los 23 departamentos representativos de Colombia. Para sustentar la relación pobreza y condiciones hidrológicas se tomó información a nivel departamental de Necesidades Básicas Insatisfechas (NBI), Producto Interno Bruto (PIB) Departamental, PIB por habitante, tasa de desempleo, tamaño de la población, extensión territorial y viviendas con acueducto.

Las variables influyentes en el entorno ambiental fueron tomadas de El Instituto de Hidrología, Meteorología y Estudios Ambientales (IDEAM) y del Informe del estado de la vigilancia de la calidad del agua para consumo humano, como es el Índice de Riesgos de la Calidad del Agua (IRCA), la proporción del área de suelos degradados por erosión (magnitud de la erosión), el porcentaje superficie cubierta por bosque, severidad de la erosión, el porcentaje de incendios, la deforestación, y por último los indicadores hídricos de oferta hídrica, demanda hídrica, índice de regulación hídrica, índice uso del agua e índice de aridez.

Con esta información se formuló un modelo de regresión a dos niveles, incluyendo como variable de respuesta el indicador de incidencia de pobreza monetaria y como variables explicativas los indicadores socioeconómicos e hidrológicos de los departamentos. En consecuencia, se realizó un modelo multinivel porque algunos indicadores hidrológicos no están en series de tiempo por departamento si no a nivel de área hidrológica, permitiendo descubrir interrelaciones entre las variables y de acuerdo con los resultados proponer los análisis estadísticos más apropiados debido a las limitaciones y ausencia de la información hídrica desagregada por departamentos. Se consideró un modelo multinivel porque algunos indicadores hidrológicos no se tienen por departamento si no a nivel de región hidrológica. Las variables del modelo son:

Variable respuesta:

- Incidencia de la pobreza monetaria.

Variables independientes a nivel departamental (nivel 1):

- Necesidades Básicas Insatisfechas (NBI).

- Tasa de desempleo.

- Índices de Riesgos de la Calidad del Agua (IRCA).

- Proporción del área de suelos degradados por erosión (magnitud de la erosión).

Variables independientes a nivel regional (nivel 2):

- Demanda hídrica. 


\section{Ćlíf 32}

Revista CIFE / ISSN: 0124-3551 e-ISSN: 2248-4914 / Bogotá-Colombia / Vol. 20 N.o 32 / enero-junio 2018 / pp. 51-77

- Oferta hídrica.

El modelo es de la forma:

$$
Y i j=\beta_{1} X_{1 i}+\beta_{2} X_{2 i}+\beta_{3} X_{3 i}+\beta_{4} X_{4}+\beta_{5} X_{5 j}+\beta_{6} X_{6 j}+\varepsilon_{i j}+\mu_{j}
$$

Donde:

$i$, es el indicador de cada departamento.

j, es el indicador de cada región.

$X_{l i}$, es el NBI de cada departamento.

$X_{2 i}$, es la tasa de desempleo de cada departamento.

$X_{3 i}$, es el IRCA de cada departamento.

$X_{4 i}$, es la magnitud de la erosión de cada departamento.

$X_{5 j}$, es la oferta hídrica de cada región.

$X_{6 j}$, es la demanda hídrica de cada región.

$\varepsilon_{i j} \sim \mathcal{N}\left(0, \sigma^{2}\right)$, es el error de los efectos aleatorios (nivel 1).

$\mu_{j} \sim \mathcal{N}\left(0, \sigma_{\mu}^{2}\right)$, es el error de los efectos fijos (nivel 2).

Posteriormente, con el fin de evaluar la evolución de la incidencia de la pobreza monetaria y teniendo información histórica desde el año 2008 hasta el 2016 de las covariables tasa de desempleo e IRCA, se realizó un modelo lineal longitudinal, esto es teniendo en cuenta las mediciones por año:

El modelo es de la forma:

$$
\text { Yit }=\beta_{0}+\beta_{1} X_{1 i t}+\beta_{2} X_{2 i t}+\varepsilon_{i t}
$$

Donde:

$i$, es el indicador de cada departamento

t, es el indicador del tiempo, desde 2008 hasta 2016

$X_{1 i t}$, es la tasa de desempleo de cada departamento para cada año

$X_{2 i t}$, es el IRGA de cada departamento para cada año

$\varepsilon_{i j} \sim \mathcal{N}\left(0, \sigma_{\varepsilon}^{2}\right)$, es el error del modelo 


\section{Análisis de resultados}

\subsection{Análisis de indicadores de pobreza}

Teniendo en cuenta el foco de estudio de la relación entre la pobreza y las condiciones hidrológicas y socioeconómicas de los 23 departamentos ${ }^{2}$ y 26 variables, en primera instancia se analizaron los distintos indicadores de pobreza reportados a nivel departamental, con el fin de seleccionar o construir un indicador que explique el nivel de pobreza para incluirlo como variable dependiente (o respuesta) en el modelo estadístico. Para la medición de la pobreza por departamento se cuentan con 7 indicadores que cuantifican algunos aspectos de la pobreza, para los cuales se realizó un análisis de correlaciones que se presenta en la siguiente tabla

Tabla 1. Matriz de correlaciones de pobreza

\begin{tabular}{|c|c|c|c|c|c|c|c|}
\hline \multicolumn{8}{|c|}{ Matriz de correlaciones } \\
\hline & 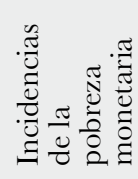 & 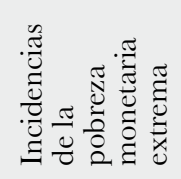 & 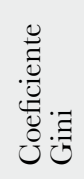 & 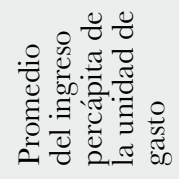 & 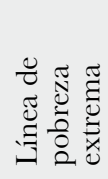 & 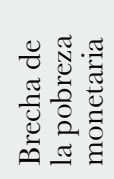 & 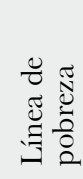 \\
\hline $\begin{array}{l}\text { Incidencias de la pobreza } \\
\text { monetaria }\end{array}$ & 1.00 & 0.33 & 0.28 & -0.10 & -0.12 & 0.28 & -0.25 \\
\hline $\begin{array}{l}\text { Incidencias de la pobreza } \\
\text { monetaria extrema }\end{array}$ & 0.33 & 1.00 & 0.75 & -0.72 & -0.64 & 0.97 & -0.72 \\
\hline Coeficiente Gini & 0.28 & 0.75 & 1.00 & -0.27 & -0.51 & 0.68 & -0.58 \\
\hline $\begin{array}{l}\text { Promedio del ingreso } \\
\text { percápita de la unidad de } \\
\text { gasto }\end{array}$ & -0.10 & -0.72 & -0.27 & 1.00 & 0.63 & -0.80 & 0.69 \\
\hline Línea de pobreza extrema & -0.12 & -0.64 & -0.51 & 0.63 & 1.00 & -0.60 & 0.95 \\
\hline $\begin{array}{l}\text { Brecha de la pobreza } \\
\text { monetaria }\end{array}$ & 0.28 & 0.97 & 0.68 & -0.80 & -0.60 & 1.00 & -0.67 \\
\hline Línea de pobreza & -0.25 & -0.72 & -0.56 & 0.69 & 0.95 & -0.67 & 1.00 \\
\hline
\end{tabular}

Fuente. Elaboración propia

De acuerdo con los resultados de la matriz de correlaciones, se realizó un Análisis de Componentes Principales (ACP) para estudiar las relaciones multivariadas existentes entre estos 7 indicadores. Del ACP se obtiene como resultado que, en los dos primeros componentes principales, se contiene el $79.3 \%$ de la varianza de todos los indicadores. Los resultados evidencian que existen correlaciones altas entre varios pares de variables, en primer lugar, la incidencia de la pobreza monetaria extrema tiene una correlación

2 Se determinaron 23 departamentos seleccionados según la muestra determinada por el DANE de la Encuesta Continua de Hogares y Gran Encuesta Integrada de Hogares, donde relaciona 24 departamentos sobre las mediciones oficiales de pobreza en Colombia, para el objeto de estudio se excluyen los datos de Bogotá. 
del $97 \%$ con la brecha de la pobreza monetaria, seguido con un $68 \%$ la correlación positiva del coeficiente Gini con la incidencia de la pobreza monetaria extrema y con la incidencia de la pobreza monetaria extrema en un $75 \%$. De igual manera, la brecha de la pobreza monetaria se correlaciona en un $97 \%$ con la incidencia de la pobreza monetaria extrema, y por último el grado de relación entre la línea de pobreza y la línea de pobreza extrema, representando un $95 \%$, validando el enfoque objetivo en la privación de necesidades básicas, limitado a la forma de vida del individuo donde una mayor vulnerabilidad en la pérdida de ingresos al interior de un hogar, no permitirá la adquisición de los satisfactores básicos mínimos para establecer una condición física aceptable y condición de vida a en el ámbito territorial.

Por otra parte, la figura 1 corresponde al componente en espacio rotado, donde se observa que los indicadores: línea de pobreza extrema, línea de pobreza y promedio de ingresos per cápita apuntan en la misma dirección hacia el primer componente, en cambio, los indicadores coeficiente Gini, incidencia de la pobreza monetaria extrema y la brecha de la pobreza monetaria también apuntan hacia el primer componente, pero en sentido inverso, lo anterior revela que estos 6 indicadores miden una misma dimensión del nivel de pobreza. Por otro lado, se observa que el componte 2, está explicado principalmente por el indicador de la incidencia de la pobreza monetaria. De acuerdo con lo anterior, se concluye que el primer componte mide los límites, brechas y desigualdad de riquezas, mientras que el segundo mide la pobreza monetaria, por lo tanto, se tomará en cuenta solo la incidencia de pobreza monetaria para estudiar la relación con las condiciones hidrológicas y socioeconómicas de los departamentos.

Figura 1. Componente de espacio rotado de pobreza

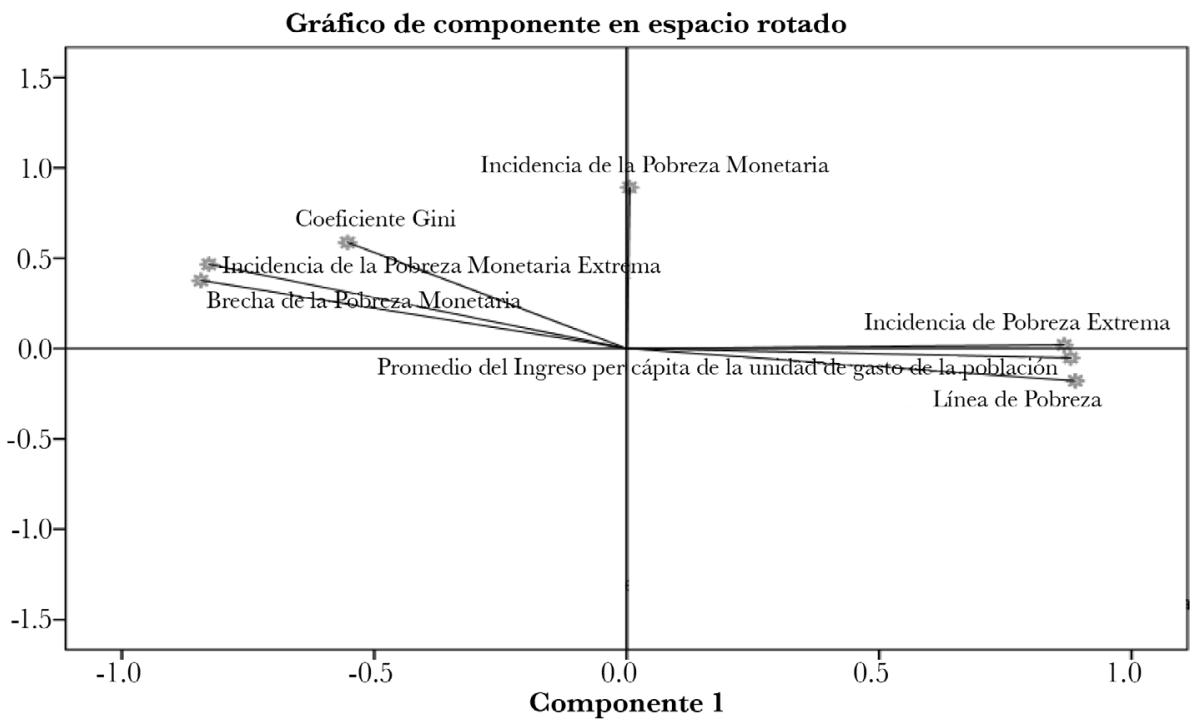

Fuente. Elaboración propia 
En la siguiente tabla se presenta la relación de la pobreza monetaria como componente principal de la pobreza, con las condiciones hidrológicas departamentales. Aquí se puede advertir que estas dos variables tienen una relación directa que es estadísticamente significativa como se muestra en el siguiente cuadro:

Tabla 2. Estimaciones de parámetros de covarianza ${ }^{a}$

\begin{tabular}{|lccc|ccc|}
\hline Parámetro & Estimación & $\begin{array}{c}\text { Error } \\
\text { estándar }\end{array}$ & Wald Z & Sig. & \multicolumn{2}{c|}{$\begin{array}{c}\text { Intervalo de } \\
\text { confianza al 90 \% }\end{array}$} \\
\hline Residuo & 11.928 & 4.217 & 2.828 & 0.005 & 6.668 & 21.337 \\
Límite & Límite \\
inferior & superior \\
\hline a. Variable dependiente: incidencia de la pobreza monetaria & & & \\
\hline
\end{tabular}

Fuente. Elaboración propia

De lo anterior se puede determinar que la varianza de los errores de los efectos aleatorios es significativa, es decir que las variables consideradas en ambos niveles del modelo sí tienen un efecto sobre la incidencia de la pobreza monetaria. La relación que existe entre la variable respuesta y las variables independientes incluidas en el modelo se evalúan mediante la estimación de los efectos fijos o parámetros $\beta \mathrm{k}$ del modelo planteado (tabla 3).

Tabla 3. Estimaciones de efectos fijosa

\begin{tabular}{|c|c|c|c|c|c|c|c|}
\hline \multirow{2}{*}{ Parámetro } & \multirow{2}{*}{ Estimación } & \multirow{2}{*}{$\begin{array}{c}\text { Error } \\
\text { estándar }\end{array}$} & \multirow{2}{*}{ gl } & \multirow{2}{*}{$\mathbf{t}$} & \multirow{2}{*}{ Sig. } & \multicolumn{2}{|c|}{$\begin{array}{c}\text { Intervalo de } \\
\text { confianza al } 90 \%\end{array}$} \\
\hline & & & & & & $\begin{array}{l}\text { Límite } \\
\text { inferior }\end{array}$ & $\begin{array}{l}\text { Límite } \\
\text { superior }\end{array}$ \\
\hline $\begin{array}{l}\text { Demanda } \\
\text { hídrica }\end{array}$ & 3.E-04 & 1.E-04 & 16 & 3.287 & .005 & 2.E-04 & 5.E-04 \\
\hline Oferta hídrica & -4.E-05 & 1.E-05 & 16 & -2.896 & .011 & $-6 . \mathrm{E}-05$ & -2.E-05 \\
\hline IRCA & 0.27 & 0.06 & 16 & 4.628 & .000 & 0.17 & 0.38 \\
\hline $\begin{array}{l}\text { Magnitud de la } \\
\text { erosión }\end{array}$ & -0.21 & 0.05 & 16 & -4.080 & .001 & -0.31 & -0.12 \\
\hline Tasa desempleo & 1.88 & 0.39 & 16 & 4.851 & .000 & 1.20 & 2.56 \\
\hline NBI & 0.78 & 0.05 & 16 & 15.752 & .000 & 0.69 & 0.86 \\
\hline
\end{tabular}


De acuerdo con las estimaciones de los efectos fijos todas las variables incluidas en el modelo son significativas, es decir, que estadísticamente están relacionadas con la incidencia de la pobreza monetaria, el tipo de relación lineal de cada variable es la siguiente:

Tabla 4. Variables

\begin{tabular}{|cc|}
\hline Variable Independiente & Tipo de Relación \\
\hline Demanda hídrica & Positiva \\
Oferta hídrica & Negativa \\
IRCA & Positiva \\
Magnitud de la erosión & Negativa \\
Tasa de Desempleo & Positiva \\
NBI & Positiva \\
\hline
\end{tabular}

Fuente. Elaboración propia

Según los resultados del modelo lineal y en cuanto a los indicadores socioeconómicos se concluye que el NBI y la tasa de desempleo tienen relación directa con el nivel de pobreza, es decir, una variación del porcentaje de necesidades básicas insatisfechas generará mayores niveles de pobreza, sucesivamente, regiones con mayor tasa de desempleo inciden en un deterioro de los ingresos, con lo que promueve una mayor vulnerabilidad a escenarios de pobreza. En cuanto a las variables hidrológicas, se debe delimitar las dificultades en los datos por departamento en la serie de tiempo para la variable magnitud del suelo, es decir, la degradación del suelo donde se encuentra una relación negativa, entre mayor es la magnitud del suelo con erosión en el departamento, menor es el nivel de pobreza; esta afirmación es contraría a lo señalado en los estados del arte, de hecho Zhao, Li, Wang y Deng (2015) y Franco (2011), correlacionan la gestión del recurso hídrico y la degradación del suelo a nivel regional con los niveles de pobreza, destacando niveles de significancia entre suelos degradados con mayores niveles de pobreza. Para Franco (2011), los componentes de un sistema complejo como: suelo, el clima, la biota, las costumbres de la población y el sistema hídrico, son determinantes dinámicas de una política pública de ordenamiento del uso del suelo y del régimen hídrico, esta condición permitirá una región estable en la sostenibilidad de la oferta hídrica y los ecosistemas en armonía con las necesidades de las comunidades.

Por el contrario, el Índice de Riesgo de la Calidad de Agua - IRCA tiene relación directa con la variable de pobreza, contando con buena información en el periodo de análisis 2008-2016, esto indica que los departamentos con mejor calidad de agua (IRCA bajo) tienen un nivel menor de pobreza que los de un IRCA alto. Este resultado contrasta con 
los diferentes estudios mencionados en el análisis teórico para validar los impactos de una eficiente gestión del agua, tanto a nivel urbano como rural en los componentes sociales de salud humana y los ecosistemas, además, la condición básica de acceso al agua por parte de un ser humano permite reducir la pobreza, pero requiere de la gestión del recurso hídrico, especialmente en el ámbito agropecuario a nivel mundial, así, Namara et al. (2010), describen cómo la gestión mejorada del agua para la agricultura puede contribuir a la reducción de la pobreza a través de varias estrategias: mejora la producción y la productividad, mejora las oportunidades de empleo y estabiliza los ingresos y el consumo y mejora el estado nutricional, la salud, la equidad social y el medio ambiente. Finalmente, Stiglitz et al. (2009), afirman de la necesidad de incorporar indicadores en la gestión del agua y pobreza, más allá de los ingresos por ser insuficiente en la medición de la calidad de vida.

Por último, se tiene que, a mayor demanda hídrica, mayor nivel de pobreza y a menor oferta hídrica menores niveles de pobreza, es decir, que en los departamentos con niveles bajos de pobreza son los que tienen mayor oferta hídrica superficial y demandan más recursos hídricos en sus actividades socioeconómicas que los departamentos con mayor pobreza. Esta condición concuerda con las conclusiones del Estudio Nacional del Agua 2014 (IDEAM, 2015), que indica que hay una distribución del agua de manera desigual para las diferentes áreas hidrográficas. En las áreas hidrográficas Magdalena-Cauca y Caribe, donde se encuentra el $80 \%$ de la población nacional y se produce el $80 \%$ del PIB Nacional, se estima que está solo el $21 \%$ de la oferta total de agua superficial.

\subsection{Evolución de la pobreza monetaria}

Frente a la evolución de la pobreza monetaria, se concluye que entre los años 2008 y 2016 tanto la tasa de desempleo como el IRCA, tienen un efecto positivo en la incidencia de la pobreza monetaria, esto indica que los departamentos con mayor riesgo en la calidad de agua y con mayor desempleo, tienden a tener una mayor incidencia en la pobreza monetaria, esta relación histórica se puede ver fácilmente en la siguiente figura 2, donde para el año 2008 la condición promedio de las tres variables evidencia la lógica de la correlación, donde a mayor incidencia en la pobreza monetaria mayor riesgo en la calidad del agua, junto con un mayor desempleo; contrario a la situación presentada en el año 2015.

Posteriormente, se construye el modelo lineal que es estadísticamente significativo, en el cual se puede interpretar que la variación de una unidad en la pobreza monetaria está determinada por una variación de 0,63 puntos en la tasa de desempleo y de 0,08 unidades en el índice de riesgo de la calidad del agua, como se muestra en la siguiente tabla: 
Figura 2. Evolución histórica de la pobreza

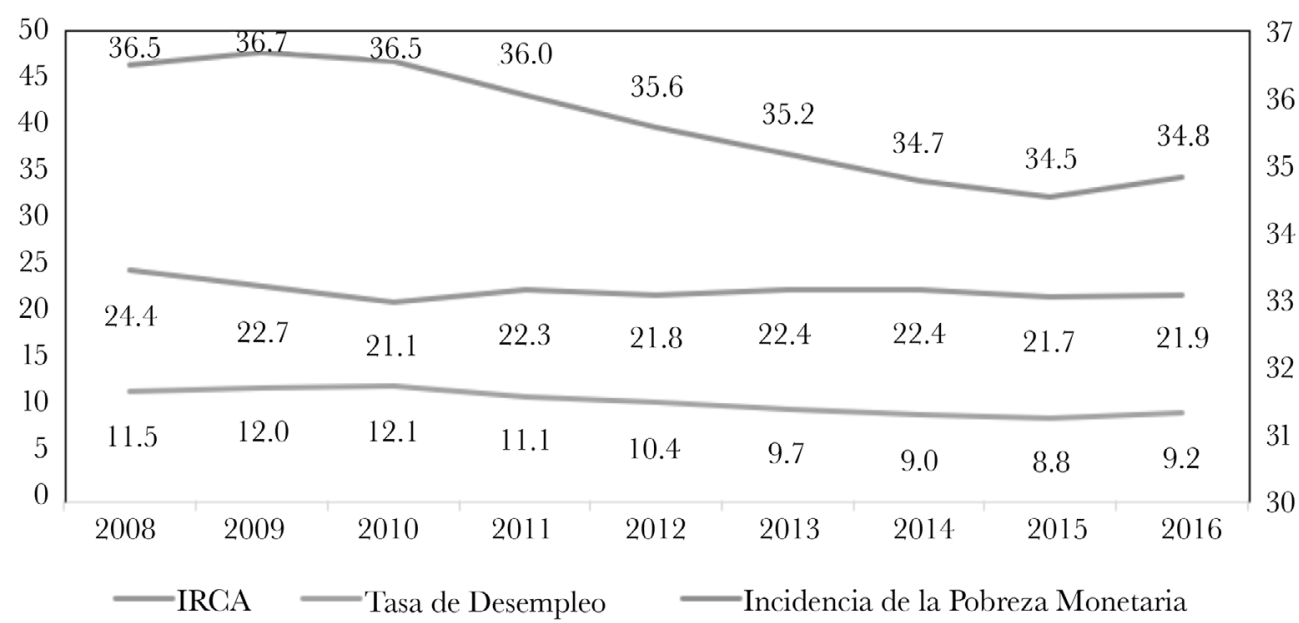

Fuente. Elaboración propia

Tabla 5. Estimaciones de efectos fijos ${ }^{\mathrm{a}}$

\begin{tabular}{|lcccccccc|}
\hline Parámetro & Estimación & $\begin{array}{c}\text { Error } \\
\text { estándar }\end{array}$ & gl & $\mathbf{t}$ & Sig. & \multicolumn{2}{c|}{$\begin{array}{c}\text { Intervalo de } \\
\text { confianza al 90 \% }\end{array}$} \\
\hline $\begin{array}{l}\text { Límite } \\
\text { inferior }\end{array}$ & $\begin{array}{c}\text { Límite } \\
\text { superior }\end{array}$ \\
\hline $\begin{array}{l}\text { Tasa de } \\
\text { desempleo }\end{array}$ & 0.63 & 0.15 & 214 & 4.232 & .000 & 0.39 & 0.88 \\
\hline IRCA & 0.08 & 0.04 & 222 & 2.070 & .040 & 0.02 & 0.14 \\
\hline
\end{tabular}

a. Variable dependiente: incidencia de la pobreza monetaria.

Fuente. Elaboración propia

Los resultados anteriores permiten plantear que, a partir del 2011, tanto la incidencia de la pobreza monetaria estimada como la tasa de desempleo en los departamentos han bajado, mientras que el IRCA en el mismo periodo se ha mantenido estable. Teniendo en cuenta que el IRCA promedio se mantiene estable a través de los años, se plantea comparar los departamentos agrupados en tres grupos, que se pueden clasificar como regiones con mayores índices de pobreza monetaria, regiones con índices promedio y regiones con menores índices de pobreza. Para esto se clasificó la variable "incidencia de la pobreza monetaria”, a través de la clasificación de clúster K-para los siguientes grupos: 
Tabla 6. Clúster de pertenencia

\begin{tabular}{|c|c|c|c|}
\hline Número del caso & Departamento & Glúster & Distancia \\
\hline 1 & Chocó & 1 & 7,650 \\
\hline 2 & Cauca & 1 & 1,750 \\
\hline 3 & La Guajira & 1 & 1,250 \\
\hline 4 & Córdoba & 1 & 2,450 \\
\hline 5 & Magdalena & 1 & 4,050 \\
\hline 6 & Sucre & 1 & 4,150 \\
\hline 7 & Nariño & 3 & 6,845 \\
\hline 8 & Huila & 3 & 6,745 \\
\hline 9 & Cesar & 3 & 6,145 \\
\hline 10 & Bolívar & 3 & 3,445 \\
\hline 11 & Caquetá & 3 &, 145 \\
\hline 12 & Norte de Santander & 3 & ,255 \\
\hline 13 & Boyacá & 3 & 1,155 \\
\hline 14 & Tolima & 3 & 2,955 \\
\hline 15 & Quindío & 3 & 4,355 \\
\hline 16 & Atlántico & 3 & 6,755 \\
\hline 17 & Caldas & 3 & 7,855 \\
\hline 18 & Meta & 2 & 2,500 \\
\hline 19 & Antioquia & 2 & 2,300 \\
\hline 20 & Risaralda & 2 & 1,800 \\
\hline 21 & Valle del Cauca & 2 & 1,600 \\
\hline 22 & Cundinamarca & 2 & 4,100 \\
\hline 23 & Santander & 2 & 4,100 \\
\hline
\end{tabular}

En Colombia, según EL Instituto de Hidrología, Meteorología y Estudios Ambientales -IDEAM (2015), el régimen hidrológico está determinado por periodos secos y húmedos, prevaleciendo una abundante oferta hídrica divididas en cinco áreas: Caribe, 
Magdalena (Cauca), Orinoco, Amazonas y Pacífico; las condiciones en estas áreas se caracterizan por ser territorialmente heterogéneas, las cuales influyen en la vulnerabilidad del sistema natural y del sistema socioeconómico. Por consiguiente, el área hidrológica de mayor oferta total nacional corresponde a la Amazonía con un porcentaje de $37 \%$, seguido del área del Orinoco con $26.3 \%$, concentrando un total de la oferta hídrica de $63.3 \%$, de la misma manera, las tres restantes áreas (Caribe, Magdalena (Cauca) y Pacífico) concentran el 36.7 \% de la oferta hídrica (IDEAM, 2015, p. 65). Así mismo, la zona hídrica de mayor rendimiento hídrico está en el Pacífico, estimado en $116 \mathrm{l} /$ skm² $^{2}$, seguido del Amazonas con 69 1/skm² y el Caribe con 56,4 1/skm² (IDEAM, 2015, p. 66).

En tal sentido, el Estudio Nacional del Agua desarrolló una estimación para los municipios más vulnerables de Colombia en relación con el abastecimiento en condiciones extremas, identificando 318 cabeceras municipales asociándolos a la distribución por área. Con rangos de valores bajos en el abastecimiento de los municipales está la cuenca del Magdalena (Cauca) con 224 cabeceras, seguida de la del Caribe (71), Orinoquía (17), Amazonía (4) y Pacífico (2) (IDEAM, 2015, p. 79). En efecto, se presenta un desbalance en la concentración del $70.7 \%$ de las fuentes abastecedoras para las 224 cabeceras municipales con una oferta hídrica del $13.4 \%$ correspondiente a Magdalena (Cauca), por el contrario, el área del Amazonas con un $37 \%$ de oferta concentra el 1.2 \%. En concordancia con la brecha existente de fuentes de abastecimiento y oferta hídrica, la demanda de agua por área hidrológica identifica que el área de Magdalena (Cauca) con el 67 \% de la demanda total de agua, seguido del Caribe (16\%), Orinoquía (12\%), Pacífico $(4 \%)$ y Amazonas (1\%); sumado a esta situación el área de Magdalena (Cauca) en la distribución de todos los sectores por uso de agua, lidera todos los sectores económicos y usos domésticos, solo con la particularidad del sector hidrocarburos liderado con mayor concentración en el área hidrológica del Orinoco (IDEAM, 2015).

En efecto, el uso de agua de las tres áreas hidrológicas con mayor presión y vulnerabilidad, tanto en la oferta como en la demanda de agua (área Magdalena (Cauca), Caribe y Pacífico), generan un principio de dependencia del recurso del agua para enfrentar la sostenibilidad económica en los principales sectores dinamizadores de la economía regional, como son en su orden de significancia: agrícola, energía hidrotermo y usos domésticos; sobre todo el área hidrológica del Magdalena (Cauca) está sometida a cambios climáticos representados en el fenómeno de El Niño donde afecta el caudal de agua. A esta situación coyuntural se suma la concentración de la población representada en un $60 \%$ de esta, asentada de zonas urbanas, lo cual establece una situación de riesgo en la gestión del recurso hídrico por enfrentar una débil disponibilidad de agua representada en $13.4 \%$ (OECD, ECLAD, 2014). Por esta razón, las evidencias en la contaminación de los cuatro ríos más importantes de Colombia, está relacionado con el río Magdalena y el río Cauca, como también el río Bogotá y Chicamocha, encontrando componentes 
de metales pesados y otros contaminantes (SIAC, 2011, citado en OECD, ECLAD, 2014, p. 37). En la tabla 6 se evidencia que el grupo 1 agrupa los departamentos con mayores niveles de pobreza, el rango 2 los de niveles de pobreza media y por último el clúster 3 los de menor nivel de pobreza monetaria, con los grupos anteriores se comparó el IRCA como índice de la calidad del agua en estos grupos:

Figura 3. Grados de relación niveles de pobreza y el IRCA

\begin{tabular}{|c|c|c|c|c|c|c|c|c|c|c|c|}
\hline & \multirow{2}{*}{ IRGA } & \multicolumn{10}{|c|}{ Año de la medición } \\
\hline & & 2008 & 2009 & 2010 & 2011 & 2012 & 2013 & 2014 & 2015 & 2016 & Total \\
\hline \multirow{4}{*}{ 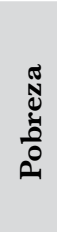 } & Alta & 29,1 & 25,7 & 23,3 & 28,4 & 26,1 & 27,6 & 27,7 & 24,2 & 28,3 & 26,7 \\
\hline & Promedio & 22,9 & 23,3 & 21,1 & 21,0 & 23,4 & 24,3 & 22,9 & 24,9 & 23,5 & 23,0 \\
\hline & Bajo & 19,0 & 17,5 & 17,7 & 14,8 & 13,7 & 12,5 & 14,1 & 14,0 & 15,0 & 15,4 \\
\hline & Total & 24,4 & 22,7 & 21,1 & 22,3 & 21,8 & 22,4 & 22,4 & 21,7 & 22,7 & 22,4 \\
\hline
\end{tabular}

Nota: La línea segmentada $a_{--.}^{-}$indica un IRCA alto y la línea continua $\square$ un IRCA bajo.

Fuente. Elaboración propia

De acuerdo con la tabla 7, se aprecia que los departamentos del grupo con mayor nivel de pobreza monetaria (alta) tienen así mismo peores indicadores de calidad del agua (IRCA), con una tendencia de mantenerse en el periodo de análisis, es decir, el año 2008 representa el mayor riesgo en la calidad de agua a nivel territorial con un 29.1 \%, dado con un nivel de pobreza alta según el primer grupo (1). Por otro lado, el año 2015 representa el periodo de bajo nivel de pobreza con los mejores indicadores de IRCA, validando una vez más la relación entre este indicador y los niveles de pobreza por departamento. De esta manera se concluye que el acceso a recursos ecosistémicos básicos hace parte de las condiciones necesarias para reducir la pobreza.

En efecto en Colombia se pueden evidenciar que las dinámicas del uso y la cantidad del agua dulce existente en la biosfera entran en conflicto por la presión antropocéntrica, evidenciando una escala de escasez del recurso natural, no solo desde la perspectiva de valor económico sino también del valor social. Por lo anterior "el acceso al agua ha sido reconocido como un derecho fundamental para una vida digna" (Guevara y Lara, 2015, p. 464), y eso implica que el deterioro de los ecosistemas y los problemas ambientales desencadenan conflictos socioambientales que tienen que ver con la tendencia degradadora de los activos ambientales para explotarlos por las comunidades más pobres para su subsistencia, al tiempo que estas perciben de manera directa el daño ambiental. Como lo plantea el Programa de Naciones Unidas para el Desarrollo (1997, p. 69) "en épocas de tensión la gente recurre en medida todavía mayor al medio natural, como 
reserva y como parte importante de la estrategia para hacer frente a la pobreza (provocando) el agotamiento y contaminación de los activos naturales".

Según la Organización para la Cooperación y el Desarrollo Económicos (OCED) "Colombia posee abundantes recursos de agua dulce, equivalentes a $49.000 \mathrm{~m} 3$ per cápita, valor muy superior al promedio de los países de América Latina y el Caribe de 7.200 m3" (IDEAM, 2010, citado en OECD, ECLAD, 2014, p. 29). Es decir, la disponibilidad de los recursos hídricos en Colombia es abundante, comparado con otros países, máxime si se considera que "cerca de un $75 \%$ de la superficie del globo está cubierta por agua, (y) menos del $1 \%$ es apta para sostener la vida humana" (Arévalo, Lozano y Sabogal, 2011, p. 103).

En este contexto, no es suficiente entender la pobreza desde la perspectiva de ingresos monetarios sin interpretar las realidades territoriales en la privación del recurso agua para satisfacer necesidades individuales y colectivas en el ser humano y otros seres vivos; así, se resalta la presencia de NBI, desempleo, IRCA y área degradada como las variables más significativas en la incidencia de la pobreza monetaria. Esta última presenta una relación negativa con la pobreza, la cual se debe profundizar en el análisis con variables que influyen en esta, como el cambio climático, desertificación derivado de la presión de ser humano y de las actividades económicas históricas de los territorios, donde se están generando condiciones de migración interna de la población. Por tanto, al reducir la productividad de los ecosistemas, los efectos en los sistemas económicos y ambientales impactan en la oferta y demanda hídrica, y de esta manera genera inestabilidad en las condiciones sociales.

\section{Conclusiones}

Se valida la relación de indicadores biofísicos e información socioeconómica que permite interpretar la relación entre las condiciones del recurso agua como determinante de pobreza a nivel territorial para determinar la vulnerabilidad de la población a nivel departamental en Colombia. En ese sentido, las relaciones entre las condiciones hidrológicas y la pobreza a nivel departamental para el período 2016, resultado del modelo lineal y en cuanto a los indicadores socioeconómicos, permiten evidenciar una relación directa de las NBI y la tasa de desempleo con el nivel de pobreza. En cuanto a las variables hidrológicas está representado en el Índice de Riesgos de la Calidad de Agua IRCA, con una relación positiva con la incidencia de la pobreza monetaria, esto indica que los departamentos con mejor calidad de agua (IRCA bajo) tienen un nivel menor de pobreza que los departamentos con un IRCA alto. Estas relaciones se caracterizan por las condiciones básicas de las actividades económicas y estilo de vida de una población con la gestión del recurso hídrico, donde se debe incorporar al análisis de pobreza, las 
condiciones hidrológicas más allá de solo el factor de acceso al recurso agua, así variables del régimen natural y de intervención antrópica, determinarán otra forma de interpretar la disponibilidad y acceso del recurso hídrico a nivel territorial.

Para futuros desarrollos de investigaciones se podría analizar la relación de pobreza con los servicios ecosistémicos, estableciendo interacciones interdisciplinares entre la economía y la ecología, para evaluar el uso que se tiene de estos servicios en la mejora de la sostenibilidad territorial y su relación con los indicadores de pobreza y desigualdad. Por tanto, se debe ampliar la recolección de información para generar sistemas de indicadores hídricos compatibles con las series de tiempo de los indicadores económicos y así ser soporte de política pública integral de los recursos naturales. De esta manera, no se limitará la relación de pobreza y agua a una condición de acceso a un recurso vital, el cual constituye un factor de libertad y desarrollo del individuo en el marco del desarrollo sostenible.

Dentro de la literatura recopilada y desarrollado el marco conceptual de la pobreza y la relación con su entorno ecológico, en especial la relación con las condiciones del recurso agua, es limitada y esto se evidencia en los análisis dominantes el enfoque objetivo y subjetivo de la pobreza por el soporte de información para su respectiva medición. En cuanto a los aspectos metodológicos la información domínate está a nivel nacional y distribuido por áreas hídricas, dificultando el análisis a nivel local, finalmente, los desarrollos científicos han contribuido al cuidado y mejoramiento de la calidad del agua, pero no se le puede asignar toda la responsabilidad del cuidado de dicho recurso a la ciencia. En realidad, la gestión del agua debe darse por un cambio en varios contextos como el social, económico y ambiental, donde se integren al modelo económico otros enfoques como puede ser la economía ecológica.

\section{Referencias}

Abadí, A. y Vitale, S. (Abril-junio, 2015). La pobreza: un mal que persiste. Revista Debates IESA, 20(2).

Alkire, S. (2011). Counting and Multidimensional Poverty Measurement. Fournal of Public Economics, 95(7-8), 476-487.

Alkire, S. y Santos, M. E. (2010). Acute Multidimensional Poverty: A New Index for Developing Countries. Human Development Researcb Paper. PNUD.

Alkire, S. y Foster, J. (2009). Counting and multidimensional poverty measurement (Working Paper 32). OPHI. 
Revista CIFE / ISSN: 0124-3551 e-ISSN: 2248-4914 / Bogotá-Colombia / Vol. 20 N.o 32 / enero-junio 2018 / pp. 51-77

Alkire, S. y James, F. (2007). Counting and Multidimensional Poverty Measurement. OPHI Working Paper Series (7). Oxford Poverty y Human Development Iniative.

Arévalo, D., Lozano, J. y Sabogal,J. (2011). Estudio nacional de huella hídrica Colombia, sector agrícola. Revista Internacional de Sostenibilidad, Tecnología y Humanismo, (6), 101-126.

Beccaria, E. y Minujin, A. (1985). Métodos alternativos para medir la evolución del tamaño de la pobreza, (documento de trabajo, 6). Buenos Aires: INDEC.

Boltvinik, J. (Abril, 1992). El método de medición integrada de la pobreza. Una propuesta para su desarrollo. Comercio Exterior, 42(4), 354-365.

Campana, M. (2014). Desarrollo humano, producción social de la pobreza y gobierno de la pobreza. Trabajo Social, (16), 79.

Carrasco, M. W. (2016). Estado del arte del agua y saneamiento rural en Colombia. Revista de Ingeniería, (44). Recuperado de http://www.redalyc.org/articulo. $\underline{\text { oa?id }=121046459008}$

Cortés, R. y Marshall, A. (1993). Política social y regulación de la fuerza de trabajo. Cuadernos Médico Sociales, (65-66), 4-32. Rosario: Centro de Estudios Sanitarios y Sociales.

Consejo Nacional de Evaluación de la Política de Desarrollo Social. (Enero-marzo, 2014). Medición Multidimensional de la Pobreza en México. El Trimestre Económico, $81(1), 5-42$.

Danani, C. y Hintze. S. (2011). Protecciones y desprotecciones: la seguridad social en la Argentina 1990-2010. Buenos Aires: Universidad Nacional de General Sarmiento - Prometeo.

Departamento Administrativo Nacional de Estadística - DANE. (2016a). 16 de los 24 departamentos estudiados redujeron la pobreza extrema en 2015 y 13 disminuyeron la pobreza monetaria. Bogotá, D. C., Colombia. Recuperado de https://www.dane.gov.co/files/ investigaciones/condiciones vida/pobreza/2015/cp pobreza departamentos $\underline{\text { R2015.pdf }}$

Departamento Administrativo Nacional de Estadística - DANE. (2016). Pobreza monetaria y multidimensional en Colombia (2015). Bogotá, D. C., Colombia. Recuperado de https://www.dane.gov.co/files/investigaciones/condiciones_vida/pobreza/bol_pobreza 15 .pdf

Deaton, A. (2001). Counting the world's poor: Problems and possible solutions. World Bank Research Observer, 16(2), 125-147. 
Domínguez, J. y Martín, A. M. (Diciembre, 2006). Medición de la pobreza: una revisión de los principales indicadores. Revista de Métodos Cuantitativos para la Economía y la Empresa, 27-66.

Donald, A. y Mottershaw, E. (2009). Poverty, inequality and buman rigbts. New York: Joseph Rowntree Foundation.

Falappa, F. y Andrenacci, L. (2009). La política social de la Argentina democrática (1983-2008). Buenos Aires: Universidad Nacional de General Sarmiento - Biblioteca Nacional.

Feres,J. C. y Mancero, X. (2001). Enfoques para la medición de la pobreza: breverevisión de la literatura. Comisión Económica para América Latina y el Caribe. Recuperado de http: / / repositorio.cepal.org/bitstream/handle/11362/4740/S01010056 es.pdf?sequence=1

Ferrer-i-Carbonell, A. (2002). Subjective questions to measure welfare and well-being: A survey, (Discussion Paper TI2002-020/3). Ámsterdam: Tinbergen Institute.

Fleury, S. (1997). Estado sin ciudadanos. Buenos Aires: Lugar Editorial.

Franco, R. A. (2011). Diseño e implementación de un modelo conceptual para la gestión integral del agua y los usos del suelo en la región de la Mojana. (Trabajo de grado maestría en Recursos Hidráulicos). Bogotá: Facultad de Ingeniería, Universidad Nacional de Colombia.

García, G. (2016). Pobreza y globalización, ¿existe correlación condicional? Una aproximación desde América latina. Globalización, Competitividad y Gobernabilidad, 10(2). Universia.

Giménez, M. y Valente, A. (Enero-junio, 2016). Una aproximación a la pobreza desde el enfoque de capacidades de Amartya Sen. Provincia, (35), 99-149.

Gordon, D. (2010). Metodología de medición multidimensional de la pobreza para México a partir del concepto de privación relativa. En M. Minor (Coord.). Medición multidimensional de la pobreza en México. México: El Colegio de México y Coneval.

Gordon, D. y Townsend, P. (2000). Measuring absolute and overall poverty. Breadine Europe: Bristol, Policy Books.

Gordon, D. y Pantazis, G. (1997). Breadline Britain in the 1990. Aldershot, Ashgate.

González, J. (2011). Utilitarismo y mediciones de pobreza. Revista de Economía Institucional, 13(25), 89-103.

Griffin, K. (1989). Alternative strategies of economic development. Londres: Macmillan. 
Revista CIFE / ISSN: 0124-3551 e-ISSN: 2248-4914 / Bogotá-Colombia / Vol. 20 N. 32 / enero-junio 2018 / pp. 51 -77

Guevara, S. y Lara, P. (2015). Agua, pobreza y uso del tiempo en México: análisis cuantitativo como sustento del diseño de una política pública de doble dividendo. Revista Nova Scientia, 15(3), 462-481.

Instituto de Hidrología, Meteorología y Estudios Ambientales - IDEAM (2015). Estudio Nacional del Agua 2014. Bogotá, D. C., Colombia: Edición Instituto de Hidrología, Meteorología y Estudios Ambientales.

Jouravlev, A. (2011). Importancia de los recursos hidricos para el desarrollo socioeconómico de la región (legislación hídrica en la adaptación al cambio climático). Presented during the Workshop: definición de prioridades de investigación económica sobre la relación entre cambio climático y agua en la región. Panamá. Recuperado de http://www.eclac.cl/drni/ noticias/noticias/8/44648/Panama 3009 2011.pdf

Macías, A. (2014). Crecimiento, desigualdad y pobreza: estado de la cuestión. Revista de Economía Institucional, 16(31).

Namara, R. E., Hanjra, M. A., Castillo, G. E., Ravnborg, H. M., Smith, L. y Van Koppen, B. (2010). Agricultural water management and poverty linkages. Agricultural Water Management, 97(4), 520-527. doi:10.1016/j.agwat.2009.05.007

OECD, ECLAC (2014). OECD Environmental Performance Revieres: Colombia 2014. OECD Publishing.

Offe, C. (1990). Contradicciones en el estado de bienestar. Madrid: Alianza.

Organización de las Naciones Unidas para la Educación, la Ciencia y la Cultura Unesco. (2015). Informe de las Naciones Unidas sobre los recursos hidricos en el mundo 2015. Agua para un mundo sostenible, datos y cifras, programa mundial de evaluación de los recursos hídricos. Colombella, Perusa, Italia: Secretaría del Programa Mundial de Evaluación de los Recursos Hídricos. División de Ciencias del Agua. Recuperado de http:// unesdoc.unesco.org/images/0023/002322/232273s.pdf

Ortiz, J. y Ríos Bolívar, H. (2013). La pobreza en México, un análisis con enfoque multidimensional. Análisis Económico, 28(69).

Ortega, J., Puello, E. y Valencia, N. (2014). Pobreza rural y políticas neoliberales: Un caso por resolver en Montería-Córdoba-Colombia. Investigación y Desarrollo, 22(2).

Pérez, Z. (2011). Desigualdad, pobreza y desarrollo. El reto de la justicia distributiva en el pensamiento de John Rawls. Anuario filosófico, 44(2), 305-334. 
Pinzón, G. (2017). Factores asociados a la pobreza subjetiva en Colombia: un estudio desde el enfoque de las capacidades y la economía de la felicidad. Revista Desarrollo y Sociedad, Bogotá, (78), 11-57.

Programa de las Naciones Unidas para el Desarrollo - PNUD. (2018). Objetivos de Desarrollo Sostenible. Colombia. Recuperado de http://www.co.undp.org/content/colombia/es/home/sustainable-development-goals.html

Programa de Naciones Unidas para el Desarrollo - PNUD (1997). Informe sobre Desarrollo Humano. Madrid: Ediciones Mundi-Prensa.

Ravallion, M. (2001). Growth, inequality and poverty: looking beyond averages. World development, 29(11), 1803-1815.

Ravallion, M. (2011). On Multidimensional Indices of Poverty, (Policy Research Working Paper 5580). Washington: World Bank.

Ravallion, M. (2012). Poor, or just feeling poor? On using subjective data in measuring poverty, (Policy Research Working Paper 5968). Washington: World Bank.

Ravallion, M., Chen., S. y Sangraula, P. (2009). Dollar a Day Revisited. Economic Review, 23, 163-184. Washington: World Bank.

Ray, D. (1999). Desarrollo Económico. España: Antoni Bosch.

Rivera, I. (2012). Microeconomía de la pobreza: el caso del Perú. Economía, 35(69), 9.

Rodrik, D. (2012). The Globalization Paradox: Democracy and the Future of the World Economy. New York \& London: Norton \& Company,

Roemer, J. E. (1998). Equality of Opportunity. Londres: Harvard University Press.

Ros, J. (2004). La teoría del desarrollo y la economía del crecimiento. México D. F.: Fondo de Cultura Económica.

Rodríguez, J. y Sánchez, A. (2017). TIC y pobreza en América Latina. Íconos-Revista de Ciencias Sociales, (57), 141-160.

Sachs, J. (2005). El fin de la pobreza. Barcelona: Debate.

Sánchez, T. (Enero-junio, 2015). Descomposiciones de los cambios en la pobreza en Colombia 2002-2012. Revista Desarrollo y Sociedad, (75), 349-398.

Sen, A. (2000). Desarrollo y libertad. (octava edición). Bogotá: Planeta. 
Revista CIFE / ISSN: 0124-3551 e-ISSN: 2248-4914 / Bogotá-Colombia / Vol. 20 N.o 32 / enero-junio 2018 / pp. 51-77

Sen, A. (Marzo, 1999). Invertir en la infancia: su papel en el desarrollo. (Ponencia presentada en las conferencias magistrales: romper el ciclo de la pobreza, invertir en la infancia). París.

Sen, A. (1996). Capacidad y bienestar. En: M. Nussbaum y S. Amartya (Comps.). La calidad de vida (pp. 54-83). México: FCE.

Sen, A. (1993). Capability and Well-Being? In M. Nussbaum y S. Amartya (Comps.), Quality of Life. Oxford: Clarendon Press.

Sistema de Información Ambiental de Colombia - SIAC (2011). Informe del Estado del Ambiente y de los Recursos Naturales Renovables de Colombia, Bogotá. Instituto de Hidrología, Meteorología y Estudios Ambientales (IDEAM). Instituto de Investigación de Recursos Biológicos Alexander von Humboldt (IAvH). Instituto de Investigaciones Marinas y Costeras (INVEMAR). Instituto Amazónico de Investigaciones Científicas (SINCHI). Instituto de Investigaciones Ambientales del Pacífico (IIAP).

Sen, A. (1992). Inequality Reexamined. Nueva York: Oxford University Press.

Sen, A. (1987). The Standard of Living. In G. Hawthorn (Gomp.). The Standard of Living. Cambridge: University Press.

Sen, A. (1985a). Commodities and Capabilities. Amsterdam: Elsevier.

Sen, A. (1985b). Well-being, Agency and Ereedom: The Dewey Lectures 1984. The Journal of Philosophy, 82, 169-221.

Sen, A. (1980). Equality of What? S. McMurrin (Comp.). The Tanner Lectures on Human Values. Salt Lake City: University of Utah Press.

Soldano, D. y Andrenacci, L. (Ed.). (2006). Aproximación a las teorías de la política social a partir del caso argentino. Problemas de política social en la Argentina contemporánea, (pp. 17-79). Buenos Aires: Prometeo.

Spicker, A. y Gordon, D. (2009). Pobreza: un glosario internacional, (primera edición). Buenos Aires: Consejo Latinoamericano de Ciencias Sociales - CLACSO.

Stiglitz, J., Amartya, S. y Fitoussi, J. P. (2009). Informe de la comisión sobre la medición del desarrollo económico y del progreso social. Recuperado de http://www.ambafrance-es.org/ $\underline{\mathrm{IMG} / \mathrm{pdf} / \text { Commission Stiglitz ES.pdf }}$

Stiglitz, J., Sen, A. y Fitoussi, J. P. (2010). Report by the Commission on the Measurement of Economic Performance and Social Progress, IEP. Paris. 
Townsend, P. (1979). Poverty in the United Kingdom. Londres, Allen Lane: Penguin Books.

Veit, J. (1998). Setting adequacy standards. How governments define.

Zhao, P., Li, L., Wang, L. y Deng, C. (2015). Spatial distributions of national poor counties and soil water erosion in China. Fresenius Environmental Bulletin, 24(12a), 4408-4415. 